Results 114 patients were identified. 11 patients transferred care and were excluded. 91/103 (88.3\%) received maintenance ( $>6$ weeks) treatment. $47.3 \%$ had discontinued treatment while $52.7 \%$ remained on treatment. The median length of treatment was 73 (range 8-329) weeks. Only 23.3\% (10/43) discontinued infliximab due to clinical remision with $34.9 \%$ (15/43) stopping because of complications and $39.5 \%(17 / 43)$ due to loss of response, surgery or death. The median course of treatment for those continuing on infliximab was 101 (range 8-329) weeks. 37 patients (21 female, mean age 40 years) were on maintenance infliximab treatment for over 1 year. $73 \%$ of these patients were on combined treatment with an immunomodulator and $37.8 \%(14 / 37)$ had required dose escalation or a reduction in dose interval. In patients continuing treatment for over 1 year, the median FC was $184 \mu \mathrm{g} / \mathrm{g}$ (range 30-9000) with a median reduction in FC level post-treatment of $416 \mu \mathrm{g} / \mathrm{g}$ (range $-3000-7086$ ) and a median HBI of 4 (range $0-16$ ). $57.1 \%$ could be defined as being in clinical remission with $\mathrm{HBI}<5$.

Conclusion In our large cohort of CD patients, few patients discontinued infliximab due to clinical remission. In those continuing infliximab for over 1 year the median FC was low, suggesting good control of inflammation, and the majority of patients were in clinical remission as defined by a $\mathrm{HBI}<5$. These results support the efficacy of infliximab as maintenance therapy in $\mathrm{CD}$ but suggest that despite evidence of clinical remission the majority of patients continue therapy.

Disclosure of Interest None Declared.

\section{PWE-075 ASSOCIATION BETWEEN THIOPURINE USE AND NON-MELANOMA SKIN CANCERS IN PATIENTS WITH INFLAMMATORY BOWEL DISEASE: A META-ANALYSIS}

doi:10.1136/gutjnl-2013-304907.364

1."J Ariyaratnam, 'V Subramanian. 'Department of Gastroenterology, Leeds Teaching Hospitals, Leeds, UK

Introduction Thiopurines are the mainstay of treatment for patients with inflammatory bowel disease (IBD). Thiopurine therapy increases the risk of non-melanoma skin cancers (NMSC) in solid organ transplant patients. The data on NMSC in patients taking thiopurines for IBD is conflicting.

Methods We searched electronic databases (PubMed, OVID, the Cochrane library, EMBASE and CINAHL) for full journal articles reporting on the risk of developing NMSC in patients taking thiopurines for IBD and hand searched the reference lists of all retrieved articles. Pooled adjusted hazard ratios and $95 \%$ confidence intervals were determined using a random effects model. Publication bias was assessed using Funnel plots or Egger's test for regression asymmetry. Heterogeneity was assessed using Cochran's $Q$ and the $I^{2}$ statistic.

Results A total of 8 studies involving 60,351 patients provided data on the risk of developing NMSC in patients with IBD on thiopurines. The pooled adjusted hazard ratio of developing NMSC after exposure to thiopurines in patients with IBD was $2.275(95 \%$ CI 1.502 to 3.446$)$. There was significant heterogeneity ( $\left.I^{2} 76 \%\right)$ between the studies, but no evidence of publication bias $(P=0.15$, intercept $=-6.7$ and $95 \% \mathrm{CI}:-17.2$ to 3.7$)$. Meta regression analysis suggested that the population studied (hospital based versus population based) and duration of follow up (> 3 years) were the major contributors to the heterogeneity. Grouping studies according to population studied and also duration suggests that the risk was much higher in hospital based and shorter duration studies (Table). Conclusion The risk of developing NMSC in patients with IBD on thiopurines is only modestly elevated. This effect loses significance when studies with less than 3 years follow-up are excluded. The difference in pooled risk between population based and hospital based studies suggests the possibility that ascertainment bias could have contributed to this increased risk. Use of thiopurines to treat IBD should not be limited by this marginally increased risk of NMSC.

Disclosure of Interest None Declared.

\section{Abstract PWE-075 Table}

\begin{tabular}{lll}
\hline & Pooled Hazard Ratio & 95\% Confidence Intervals \\
\hline All Studies & 2.275 & $1.502-3.446$ \\
Follow-up $<3$ years & 2.869 & $2.017-4.080$ \\
Follow-up $>$ 6 years & 1.876 & $0.868-4.056$ \\
Population based & 1.828 & $1.196-2.795$ \\
Hospital based & 7.217 & $3.082-16.898$ \\
\hline
\end{tabular}

actions between microbial antigens and the mucosal immune system. In IBD, epithelial barrier function is impaired with altered expression of tight junctions. We aimed to assess epithelial tight junction expression and mucosal cytokines in acute and chronic pouchitis and non-inflamed pouches of patients with ulcerative colitis. 